\title{
The puzzle of lactational bone physiology: osteocytes masquerade as osteoclasts and osteoblasts
}

\author{
Brittany A. Ryan and Christopher S. Kovacs \\ Faculty of Medicine, Endocrinology, Memorial University of Newfoundland, Health Sciences Centre, Newfoundland and Labrador, Canada.
}

\begin{abstract}
Lactation is a unique period in which the maternal skeleton acts as a storehouse to provide substantial calcium to milk. Women who exclusively breastfeed lose an average of $\mathbf{2 1 0} \mathbf{~ m g}$ of calcium per day, which doubles or triples with twins and triplets. Data from rodent and clinical studies are consistent with skeletal calcium being released to provide much of the calcium needed for milk production. This is programmed to occur independently of dietary calcium intake or intestinal calcium absorption, which remains at the prepregnant rate in breastfeeding women. After weaning, the skeleton is restored to its prior mineralization and strength, but the factors that regulate this remain to be elucidated.
\end{abstract}

maintained through compensatory increases in intestinal calcium absorption $(1,6)$. A calcium-restricted diet or larger litter sizes cause greater losses of skeletal mineral content and further reductions in bone strength, whereas a calcium-enriched diet and smaller litters have the opposite effects $(1,7)$. It is only when skeletal resorption is blocked and calcium intake is simultaneously restricted that maternal mineral homeostasis is substantially impaired, leading to hypocalcemic death $(1,6)$. This confirms the idea that the programmed production of milk can overwhelm the mother's ability to maintain her serum calcium concentration (milk fever in cows).

\section{Effects of lactation on the maternal skeleton}

The maternal skeleton is borrowed from during lactation to provide much of the calcium that ends up in breast milk (1). Women who exclusively breastfeed (that is, all of the infant's nutrition comes from breast milk) lose $210 \mathrm{mg}$ of calcium daily; nursing twins or triplets causes a respective doubling or tripling of the amount of calcium lost to milk. Nursing a singleton exclusively for three to six months leads to a $5 \%-10 \%$ reduction in lumbar spine areal bone mineral density (aBMD), as assessed by dual x-ray absorptiometry (DXA). During this time, the appendicular skeleton loses half that amount or less. Clinical data are consistent with skeletal calcium being released during lactation to provide much of the calcium needed for milk production, including higher milk output being correlated with a greater loss of $\operatorname{BMD}(1,2)$. This process is programmed to occur independently of dietary calcium intake or intestinal calcium absorption, which remains at the prepregnant rate in breastfeeding women (1). Randomized interventions and observational studies have shown that high and low dietary calcium intakes do not alter the loss of aBMD during lactation $(1,3,4)$.

Studies of rodents have contributed significantly to our knowledge about the effects of lactation on the maternal skeleton. Rodents face a proportionately higher demand to supply calcium because of larger litter sizes and the relatively short duration of lactation (approximately 21 days). As a result, they require both upregulated intestinal calcium absorption and resorption of skeletal mineral content. This typically leads to a $25 \%-35 \%$ reduction in skeletal ash weight, ash calcium, and bone mineral content by DXA (1). Moreover, the rodent skeleton also exhibits losses of up to $60 \%$ in strength and stiffness of the vertebrae, femora, and tibiae (1). The effects of lactation are not uniform; instead, resorption is much more pronounced in the trabecula-rich spine than in the appendicular skeleton $(1,5)$. The relative contributions of dietary and skeletal mineral content to milk production have been shown in several ways. When skeletal resorption is blocked (e.g., with bisphosphonates, calcitonin, or denosumab), maternal mineral homeostasis and milk content are
Related Article: p. 3058

\section{How does the skeleton lose calcium to the milk?}

The first clearly defined mechanism is increased osteoclast-mediated bone resorption. Histomorphometric analyses of bone from lactating rodents and primates have revealed increased osteoclast numbers and activity, with erosion of trabecular bone and loss of trabecular numbers (1). Although such studies have not been completed in breastfeeding women, a marked increase in bone resorption markers such as C-telopeptide suggests that osteoclast numbers and activity are increased (1), whereas high-resolution peripheral quantitated CT (HR-pQCT) of the distal tibia and radius has shown the results of increased osteoclast activity, including trabecular thinning and loss and increased cortical porosity $(8,9)$.

A second mechanism of skeletal mineral loss during lactation is osteocytic osteolysis - a process by which osteocytes function like osteoclasts to resorb mineral from their surroundings $(10,11)$. The ability of osteocytes to do this was first discovered in 1881 by Rigal and Vignal, who observed widened lacunae around osteocytes (12). This was followed by von Recklinghausen's hypothesis that during times of reduced mineral availability, osteocytes digest the perilacunar matrix (13). The theory did not gain much attention or support at that time. Much later, in the 


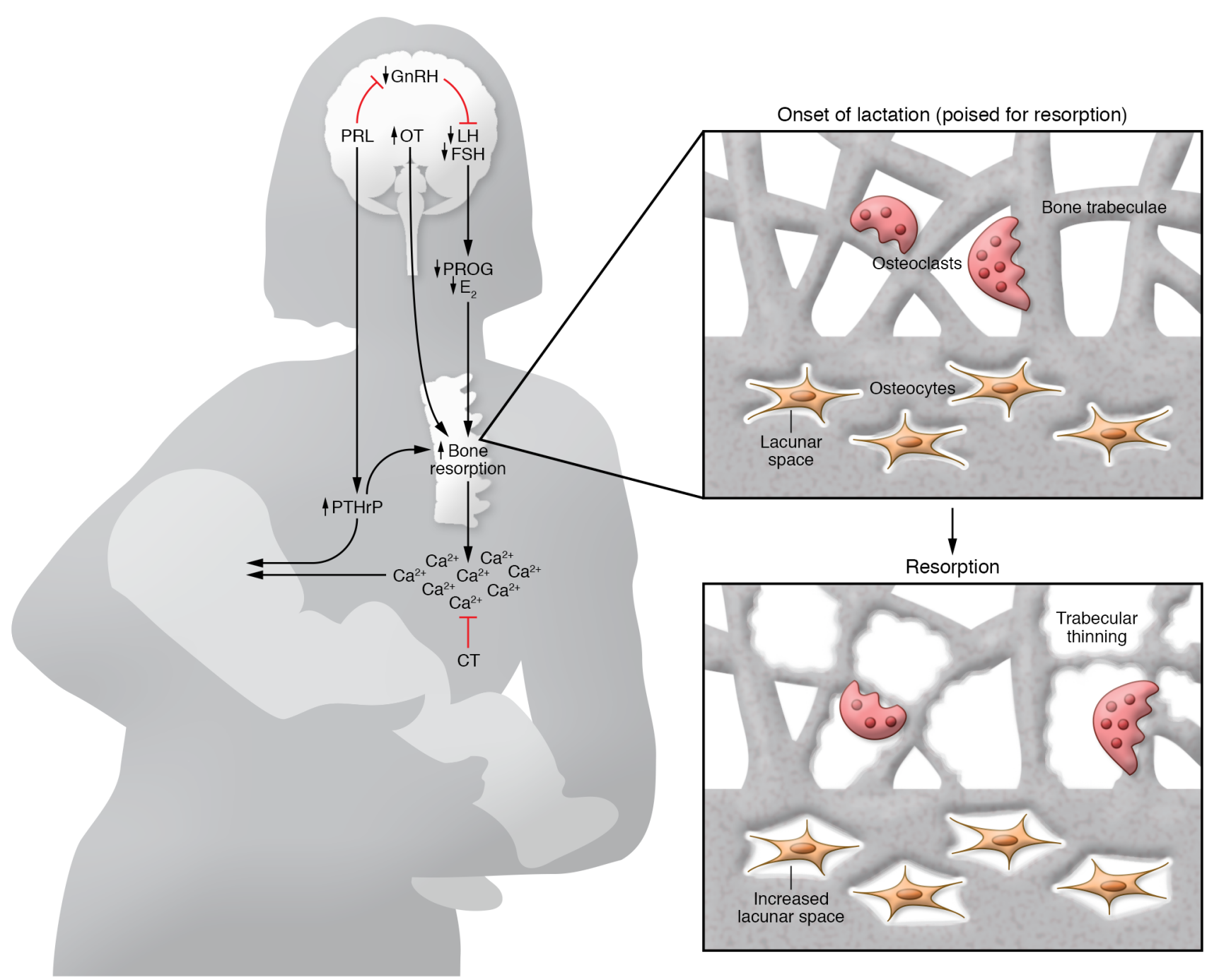

Figure 1. Breast-brain-bone circuit controls skeletal resorption during lactation. Suckling and prolactin (PRL) both inhibit the hypothalamic gonadotropin-releasing hormone $(\mathrm{GnRH})$ pulse center, which in turn suppresses the gonadotropins (luteinizing hormone [LH] and follicle-stimulating hormone $[\mathrm{FSH}]$ ), leading to low levels of the ovarian sex steroids (estradiol $\left[\mathrm{E}_{2}\right]$ and progesterone [PROG]). PRL may also have direct effects on its receptor in bone cells. PTHrP production and release from the breasts is stimulated by suckling, PRL, low estradiol, and the calcium receptor. PTHrP enters the bloodstream and combines with systemically low estradiol levels to markedly upregulate bone resorption and (at least in rodents) osteocytic osteolysis. These activities release calcium and phosphate, which reach the breast ducts and are actively pumped into the breast milk. PTHrP also passes into milk at high concentrations, but whether swallowed PTHrP plays a role in regulating calcium physiology of the neonate is uncertain. In addition to stimulating milk ejection, oxytocin (OT) may directly affect osteoblast and osteoclast function. Calcitonin may inhibit skeletal responsiveness to PTHrP and low estradiol. The inset at the top right depicts a cross-section of cortical and trabecular bone from a vertebral body at a micron level of magnification. At the onset of lactation, multinucleated osteoclasts and osteocytes are poised to resorb mineral from bone. The inset at the bottom right depicts progressive trabecular thinning by osteoclasts, whereas osteocytic osteolysis leads to a progressive increase in the lacunar area. The two processes contribute to increased cortical porosity.

1960s, Belanger rediscovered this phenomenon and called it "osteocytic osteolysis." He demonstrated in rodents that osteocytic osteolysis can be suppressed by calcitonin or a high-calcium diet and can be increased by a low-calcium diet, treatment with parathyroid hormone (PTH), or pregnancy (14, 15). Unfortunately, osteocytic osteolysis continued to be largely disbelieved and disregarded. The laboratory of Lynda Bonewald rediscovered it and, with modern techniques, demonstrated that osteocytes resorb mineral and proteinaceous matrix from their surrounding lacunae and express osteoclastrelated genes and enzymes (including cathepsin K; refs. 16, 17). They reconfirmed that this occurs during lactation in mice but not when osteocytes lack the PTH receptor $(11,16,17)$. Despite this rigorous work, osteocytic osteolysis has continued to be looked upon with skepticism.

The research from the laboratory of Roland Baron and colleagues reported in this issue of the JCI (18) convincingly shows that expression of cathepsin $\mathrm{K}$ by osteocytes is not trivial but is functionally important, since selective ablation of the cathepsin K gene from osteocytes blunted lacunar resorption and mineral loss during lactation. Moreover, the effects were more widespread, leading to prevention of the normal lactation-induced increase in osteoclast numbers and function and, in turn, reduced osteoclast-mediated bone resorption. There was little in the way of off-target ablation of cathepsin $\mathrm{K}$, and 
specifically not in osteoclasts, to explain the lack of increase in osteoclast numbers. As the authors suggest, osteocytes may communicate with osteoclasts indirectly, with matrix-related signals such as TGF- $\beta$ escaping into the circulation during osteocytic osteolysis to trigger the formation and recruitment of osteoclasts. Without osteocytic osteolysis, those signaling molecules remain bound in the osteocytic lacunae.

This work also reaffirms the ability of the rodent to increase absorption of calcium from the intestine when the skeleton is unable to provide what is needed; consequently, milk calcium content remains normal.

\section{What hormones regulate lactational bone loss?}

Two systemic factors related to lactational bone loss are low estradiol and increased parathyroid hormone-related protein (PTHrP); these and other interacting hormones are depicted in Figure 1. Low estradiol and PTHrP synergistically stimulate osteoclast-mediated bone resorption and osteocytic osteolysis and thus enable provision of calcium to the milk. In clinical studies, higher circulating PTHrP levels correlated with a greater lactational decrease in maternal aBMD at the lumbar spine and femoral neck (19). On occasion, the increase in PTHrP during lactation has caused more marked skeletal resorption and symptomatic hypercalcemia, a condition called pseudohyperparathyroidism, which typically resolves at weaning (1). A rodent model confirmed the functional importance of PTHrP: deletion of the gene from mammary tissue resulted in blunting of lactational bone loss (20).

\section{Recovery after weaning: an important avenue for future research}

After the baby is weaned, there is a reverse uncoupling of bone turnover to favor net bone formation. Within 24 hours of weaning in rodents, widespread apoptosis of osteoclasts occurs (21), followed by a surge in osteoblast numbers and function $(21,22)$, whereas osteocytes express osteoblast-specific genes and restore mineral to their lacunae (11). Six to twelve months after weaning, aBMD of the lumbar spine and hip of women has returned to prepregnancy values or better (1). The same occurs in rodents within two to four weeks, as demonstrated by ash weight measurements, DXA, and histomorphometry $(1,7,23)$. But within appendicular sites in women and rodents, skeletal recovery varies from complete restoration to persistent microarchitectural changes (largely reduced trabecular numbers and thickness and increased cortical porosity) (1, 5, 8, 9). Despite any permanent changes, bone strength appears to return to normal. In the long term, dozens of retrospective studies (1) and now two large prospective studies $(24,25)$ have demonstrated that parity and lactation do not increase the risk of fragility fractures or low BMD. In rodents, mechanical properties such as breaking strength rapidly return to prepregnancy values $(1,5,23)$.

What signals osteoclast apoptosis? What initiates and stimulates skeletal recovery? Are there signals in the bone matrix? Perhaps in the mammary tissue?

The known calciotropic hormones are not required for post-weaning skeletal recovery in rodents $(1,7,23)$; beyond this, much remains to be elucidated. This is an otherwise unprecedented interval, since most causes of bone loss in adults (such as menopause or extended weightlessness) are followed by only slow or partial recovery after the insult is removed (1). A better understanding of the physiology of bone loss and restoration during reproductive cycles may lead to the identification of new signaling molecules, which in turn could become novel treatments for bone loss from osteoporosis or prolonged space flight. Baron and colleagues have solidified the framework through which osteocytic osteolysis occurs and have added one more piece to the puzzle of how the skeletal cupboard is borrowed from and then replenished after lactation.

Address correspondence to: Christopher Kovacs, Health Sciences Centre, 300 Prince Philip Drive, St. John's, Newfoundland and Labrador, A1B 3V6 Canada. Phone: 709.864.3847; Email: ckovacs@mun.ca.

1. Kovacs CS. Maternal mineral and bone metabolism during pregnancy, lactation, and post-weaning recovery. Physiol Rev. 2016;96(2):449-547.

2. Laskey MA, et al. Bone changes after 3 mo of lactation: influence of calcium intake, breast-milk output, and vitamin D-receptor genotype. Am J Clin Nutr. 1998;67(4):685-692.
3. Cross NA, Hillman LS, Allen SH, Krause GF. Changes in bone mineral density and markers of bone remodeling during lactation and postweaning in women consuming high amounts of calcium. JBone Miner Res. 1995;10(9):1312-1320.

4. Kalkwarf HJ, Specker BL, Bianchi DC, Ranz J, Ho M. The effect of calcium supplementation on bone density during lactation and after weaning. N Engl J Med. 1997;337(8):523-528.

5. Liu XS, Ardeshirpour L, VanHouten JN, Shane $\mathrm{E}$, Wysolmerski JJ. Site-specific changes in bone microarchitecture, mineralization, and stiffness during lactation and after weaning in mice. J Bone Miner Res. 2012;27(4):865-875.

6. Ardeshirpour L, et al. OPG Treatment prevents bone loss during lactation but does not affect milk production or maternal calcium metabolism. Endocrinology. 2015;156(8):2762-2773.

7. Kirby BJ, Ma Y, Martin HM, Buckle Favaro KL, Karaplis AC, Kovacs CS. Upregulation of calcitriol during pregnancy and skeletal recovery after lactation do not require parathyroid hormone. J Bone Miner Res. 2013;28(9):1987-2000.

8. Brembeck P, Lorentzon M, Ohlsson C, Winkvist A, Augustin H. Changes in cortical volumetric bone mineral density and thickness, and trabecular thickness in lactating women postpartum. J Clin Endocrinol Metab. 2015;100(2):535-543.

9. Bjørnerem $\AA$, et al. Irreversible deterioration of cortical and trabecular microstructure associated with breastfeeding. J Bone Miner Res. 2017;32(4):681-687.

10. Teti A, Zallone A. Do osteocytes contribute to bone mineral homeostasis? Osteocytic osteolysis revisited. Bone. 2009;44(1):11-16.

11. Wysolmerski JJ. Osteocytes remove and replace perilacunar mineral during reproductive cycles. Bone. 2013;54(2):230-236.

12. Rigal A, Vignal W. Recherches expérimentales sur la formation du cal et sur les modifications des tissus dans les pseudoarthroses. Arch Physiol. 1881;Ser. II(8):419-458.

13. Von Recklinghausen F. Untersuchungen über Rachitis und Osteomalacie. Jena, Germany: Verlag von Gustav Fischer; 1910.

14. Bélanger LF. Osteocytic osteolysis. Calcif Tissue Res. 1969;4(1):1-12.

15. Belanger LF, Robichon J, Migicovsky BB, Copp DH, Vincent J. In: Sognnaes RF ed. Mechanisms of Hard Tissue Destruction. Washington, DC, USA: American Association for the Advancement of Science; 1963:531-556.

16. Qing H, et al. Demonstration of osteocytic perilacunar/canalicular remodeling in mice during lactation. J Bone Miner Res. 2012;27(5):1018-1029.

17. Qing H, Bonewald LF. Osteocyte remodeling of the perilacunar and pericanalicular matrix. Int J Oral Sci. 2009;1(2):59-65.

18. Lotinun S, et al. Cathepsin K-deficient osteocytes prevent lactation-induced bone loss and parathyroid hormone suppression. JClin Invest. 2019;129(8):3058-3071.

19. Sowers MF, et al. Elevated parathyroid hormonerelated peptide associated with lactation and bone density loss. JAMA. 1996;276(7):549-554.

20. VanHouten JN, et al. Mammary-specific deletion of parathyroid hormone-related protein pre- 
serves bone mass during lactation. J Clin Invest. 2003;112(9):1429-1436.

21. Miller SC, Bowman BM. Rapid inactivation and apoptosis of osteoclasts in the maternal skeleton during the bone remodeling reversal at the end of lactation. Anat Rec (Hoboken). 2007;290(1):65-73.

22. Collins JN, et al. Lactating Ctcgrp nulls lose twice the normal bone mineral content due to fewer osteoblasts and more osteoclasts, whereas bone mass is fully restored after weaning in association with up-regulation of Wnt signaling and other novel genes. Endocrinology. 2013;154(4):1400-1413.

23. Gillies BR, et al. Absence of calcitriol causes increased lactational bone loss and lower milk calcium but does not impair post-lactation bone recovery in Cyp27b1 null mice. J Bone Miner Res. 2018;33(1):16-26.
24. Crandall CJ, et al. Associations of parity, breastfeeding, and fractures in the Women's Health Observational Study. Obstet Gynecol. 2017;130(1):171-180.

25. Cooke-Hubley S, et al. Parity and lactation are not associated with incident fragility fractures or radiographic vertebral fractures over 16 years of follow-up: Canadian Multicentre Osteoporosis Study (CaMos). Arch Osteoporos. 2019;14(1):49. 